\title{
Stability analysis of geomagnetic baseline data obtained at Cheongyang observatory in Korea
}

\author{
Shakirah M. Amran ${ }^{1,2,3}$, Wan-Seop Kim ${ }^{1,2}$, Heh Ree Cho ${ }^{1}$, and Po Gyu Park ${ }^{1,2}$ \\ ${ }^{1}$ Korea Research Institute of Standards and Science, 267 Gajeong-ro, Yuseong-gu, Daejeon 34113, Rep. of Korea \\ ${ }^{2}$ University of Science and Technology, 217 Gajeong-ro, Yuseong-gu, Daejeon 34113, Rep. of Korea \\ ${ }^{3}$ National Metrology Institute of Malaysia, Lot PT 4803 Bdr Baru Salak Tinggi, 43900 Sepang, Selangor, Malaysia \\ Correspondence to: Po Gyu Park (pgpark@kriss.re.kr)
}

Received: 18 February 2017 - Discussion started: 23 February 2017

Revised: 16 May 2017 - Accepted: 30 May 2017 - Published: 7 July 2017

\begin{abstract}
The stability of baselines produced by Cheongyang (CYG) observatory from the period of 2014 to 2016 is analysed. Step heights of higher than $5 \mathrm{nT}$ were found in $H$ and $Z$ components in 2014 and 2015 due to magnetic noise in the absolute-measurement hut. In addition, a periodic modulation behaviour observed in the $H$ and $Z$ baseline curves was related to annual temperature variation of about $20^{\circ} \mathrm{C}$ in the fluxgate magnetometer hut. Improvement in data quality was evidenced by a small dispersion between successive measurements from June 2015 to the end of 2016. Moreover, the baseline was also improved by correcting the discontinuity in the $H$ and $Z$ baselines.
\end{abstract}

\section{Introduction}

Geomagnetic observatories data are mainly used for monitoring of secular variations (Jankowski and Sucksdorff, 1996). Data with longer time series and greater absolute accuracy are very valuable for detailed monitoring of the secular variations. In order to obtain continuous and reliable geomagnetic data, relative and absolute measurements are conducted under carefully controlled conditions. Relative measurements recorded the variations of three independent components of geomagnetic field relative to baselines using a fluxgate magnetometer. Absolute measurements are conducted on a regular basis to measure magnetic direction, declination $(D)$ and inclination $(I)$. The baseline values are derived from the difference between the absolute-measurement results and the variation data provided by a fluxgate magnetometer.
Baseline values with frequent measurement points, small drift and low scatter indicate high-quality data and a good performance of the observatory (McLean et al., 2004). In addition, good baseline stability makes monitoring of secular variations more accurate (Reda et al., 2011). Baseline variations recommended by INTERMAGNET for the participating observatories are $5 \mathrm{nT} \mathrm{yr}^{-1}$ or less (St Louis, 2012). In practice, error factors affecting the absolute-measurement instrument, the magnetometer (such as temperature, pier tilts and ageing of electronics components), and the observational procedure can cause a large drift in baseline. In this study, we present the results of the stability analysis on the observed baselines obtained from the period of 2014 to 2016. Above all, the baseline data quality of the $H$ and $Z$ components was improved by correcting the step height in the baseline curves caused by artificial magnetic components in the absolute measurements. In addition, we also analysed the temperature effect observed in the baseline as well as the quality of the absolute measurements obtained at Cheongyang observatory.

\section{Observatory site and instrumentation}

Cheongyang geomagnetic observatory (IAGA code CYG; $36.370^{\circ} \mathrm{N} ; 126.854^{\circ} \mathrm{E}$; elevation $165 \mathrm{~m}$ ), South Korea, has been in operation since 2009 and gained official INTERMAGNET magnetic observatory (IMO) status in December 2013. The CYG observatory was built in a mountainous area about $5 \mathrm{~km}$ away from the main traffic road to reduce artificial magnetic noise as shown in Fig. 1. The observatory contains five huts, separated from one another by more than 


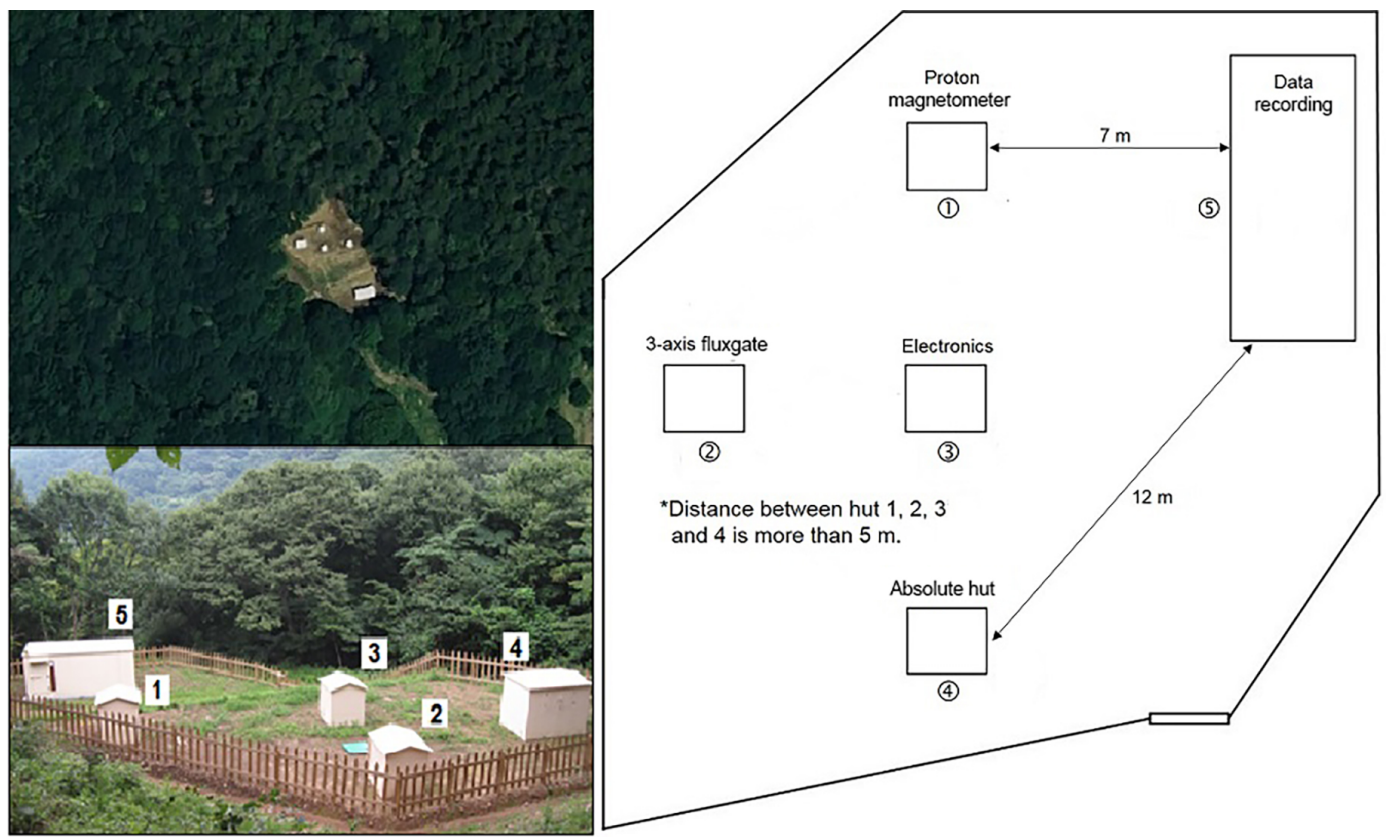

Figure 1. Location and site layout of the CYG observatory. The upper left panel shows the mountainous area where the CYG observatory is located, and the lower panel shows the observatory site. The right panel shows the layout of the observatory site. A scalar magnetometer for total field intensity measurement was installed in hut 1 . Hut 2 is used for installation of a three-axis fluxgate magnetometer, and its electronics were placed in hut 3. A pillar used for absolute measurement and which served as a reference point for total field intensity measurement was installed in hut 4 . Hut 5 is used as a control room for data acquisition and transmission to a server (Park et al., 2014).

$5 \mathrm{~m}$. In hut 1 , a scalar magnetometer was installed for measurements of the total field intensity. A three-axis fluxgate magnetometer was mounted on a marble pillar in hut 2 to measure magnetic field variations, and its electronic unit was placed in hut 3. Hut 4 contains a sturdy pillar for mounting of a fluxgate theodolite used for absolute observations. The pillar served as the reference point for the total field intensity measurement. Hut 5 is used as a control room where a computer controls data acquisition and transmission of the measured data via the Internet to a server of the Korea Meteorological Administration (KMA).

Geomagnetic variations in magnetic components $X, Y$ and $Z$ are recorded at a $1 \mathrm{~s}$ sampling rate with a resolution of $0.1 \mathrm{nT}$ by means of three-axis fluxgate magnetometer model FGE from DTU Space (Denmark). Sensors are located underground in a thermally isolated box to minimize temperature variations. The FGE electronics are placed in a separate hut to avoid magnetic interference. In addition to the fluxgate sensors, a scalar magnetometer (Overhauser effect proton precession magnetometer (PPM)) model GSM-19T from GEM Systems is independently installed for measurement of total field intensity $(F)$. The total field values are recorded every $5 \mathrm{~s}$ with a $0.1 \mathrm{nT}$ resolution.

The absolute measurements of $D$ and $I$ are conducted weekly using a non-magnetic theodolite (Zeiss 010A) with an integrated single-axis fluxgate (DTU model G). In each measurement session, four absolute measurements are per- formed on the basis of the magnetic field null method. Total field intensity difference between the absolute-measurement pillar and the PPM pillar is measured using a Cs-He standard magnetometer with a $0.1 \mathrm{nT}$ resolution (Shifrin et al., 2008). The site difference value is taken into account to correct the continuous scalar readings with reference to the magnetic field value of the absolute-measurement pillar.

The absolute measurements are processed using the Java program GDASView developed by the British Geological Survey (BGS) to derive the baseline values. Variation of the baseline values is fitted by piecewise polynomial up to third order to minimize deviation of baselines (Clarke et al., 2013).

\section{Baseline shift}

The observed baseline values of $D, H$ and $Z$ components from the measured $D, I$ and $F$ from 2014 to 2016 are shown in Fig. 2. Step heights of higher than $5 \mathrm{nT}$ can be seen in $H$ and $Z$ baselines during 2014 and 2015 . The first step with magnitude of approximately $5.2 \mathrm{nT}$ was found in $H$ on 7 July 2014. Another step with similar magnitude happened on 23 October 2014, pushing the $H$ baseline further down, and ended on 4 June 2015. On 12 June 2015, a jump of approximately $7.3 \mathrm{nT}$ occurred, bringing the baseline to a new level, and continued until the end of 2016. In addition, the $Z$ baselines follow the same trend with approximately the same magnitude. The trend indicates that an offset was in- 

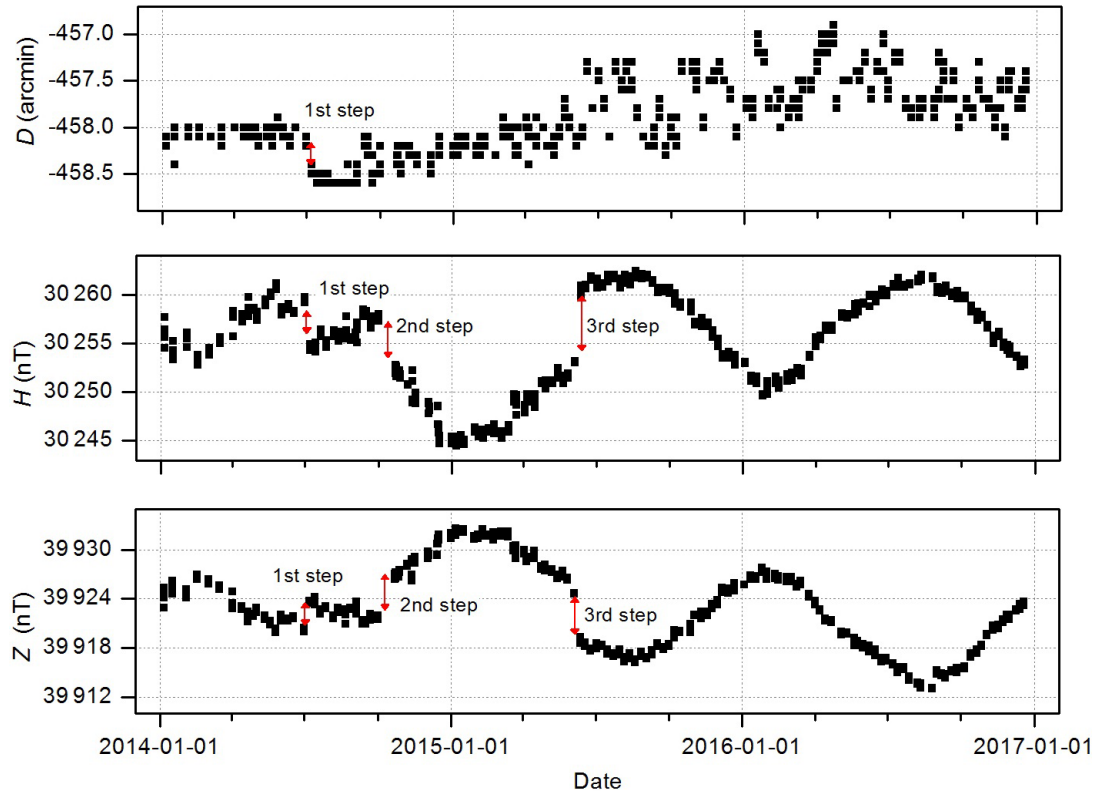

Figure 2. Observed $D, H$ and $Z$ baselines calculated from the measured $D$ (declination), $I$ (inclination) and $F$ (total field) from 2014 to 2016 . The first steps in the $D, H$ and $Z$ baselines occurred on 7 July 2014; the second and third steps were found in $H$ and $Z$ on 23 October 2014 and 12 June 2015, respectively.

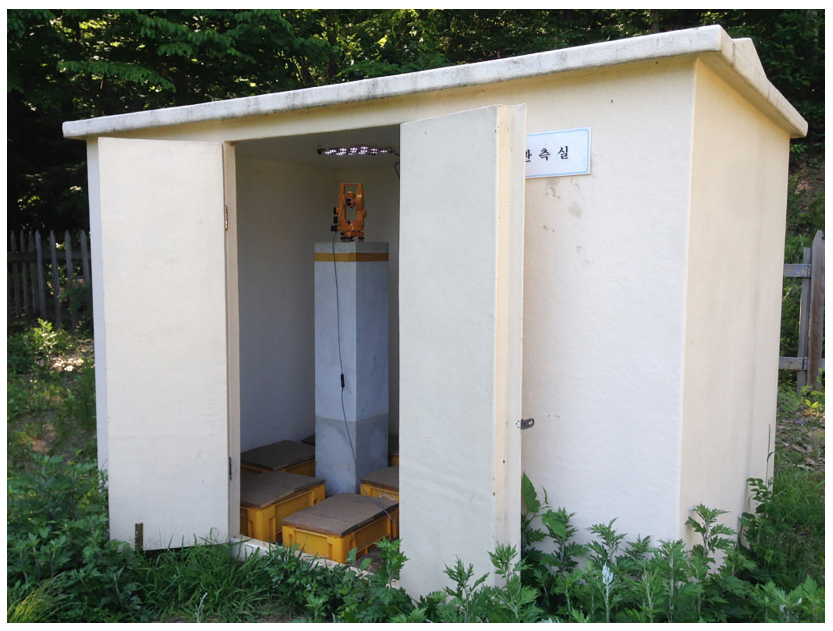

Figure 3. LED light panels installed in the absolute-measurement hut.

troduced to the baseline, which caused a baseline shift from July 2014 until June 2015. A small step can be observed in the $D$ baseline on 7 July 2014, shifting the baselines down approximately 0.4 arcmin. However, no noticeable baseline shift occurred in October 2014 or June 2015 as found in $H$ and $Z$ components.

The observatory log book in June 2014 showed that LED light panels were installed in the absolute-measurement hut on top of the absolute-measurement pillar close to the fluxgate sensor as shown in Fig. 3; they were later removed in June 2015. The period in which the LED panels were in- stalled and removed is consistent with the period when the baseline shift occurred. The magnetic part from the LED panels caused the first jump in July 2014. The LED lights may not be permanently in use because of sufficient light in the absolute-measurement hut during the summer months. However during October, when the natural light is not sufficient, the LED lights and a battery pack generate a magnetic field during the absolute measurements and cause another step in the baselines. Upon removal of the LED panels in June 2015, the offset in the baselines was removed.

To verify the consistency of the steps, the variation data was checked. However, there are no observable steps in the variation data. In addition, we compared the CYG baselines with Kakioka (KAK) observatory data for the same period as shown in Fig. 4. Although steps are noticeable in KAK baselines in October 2014, the magnitude is small: approximately $1 \mathrm{nT}$. Furthermore, no large steps can be found in KAK baselines in July 2014 or June 2015. Thus, it can be confirmed that steps that happened at CYG are due to the artificial noise which caused an error in the absolute measurements.

Adjustments were made to the baselines in order to fix the steps. For the $D$ baselines, the adjustment value was determined from the baseline difference immediately before and after the step in July 2014. The $D$ baselines from 7 July 2014 to 4 June 2015 were then adjusted. For the $H$ and $Z$ component, the baseline difference $d_{1}$ was calculated in July 2014 and was applied to the $H$ and $Z$ baselines from 7 July 2014 to 1 October 2014. Then, the next baselines differences $d_{2}$ and $d_{3}$ were calculated in October 2014 and June 2015, respectively, and the average $\left(d_{2}+d_{3}\right) / 2$ was applied from 23 Oc- 

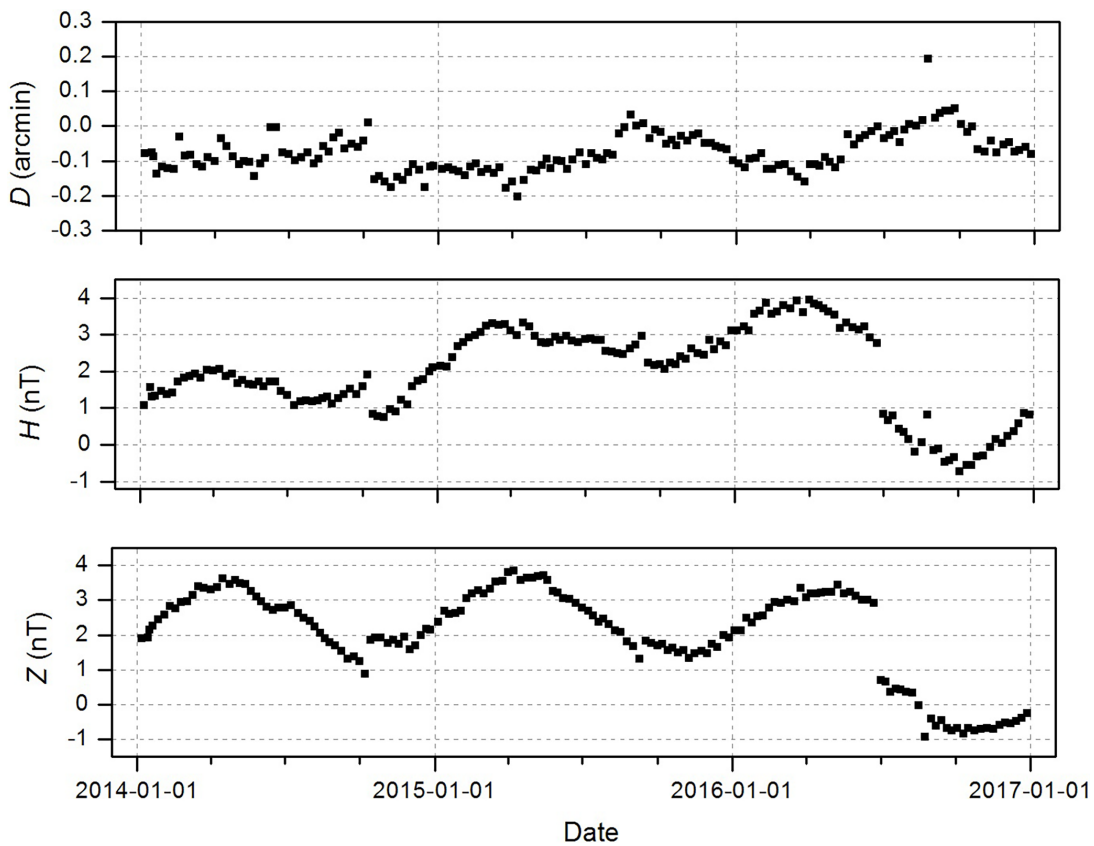

Figure 4. KAK baselines values obtained from 2014 to 2016. The data of the KAK observatory were provided by the Kakioka Magnetic Observatory, Japan Meteorological Agency.
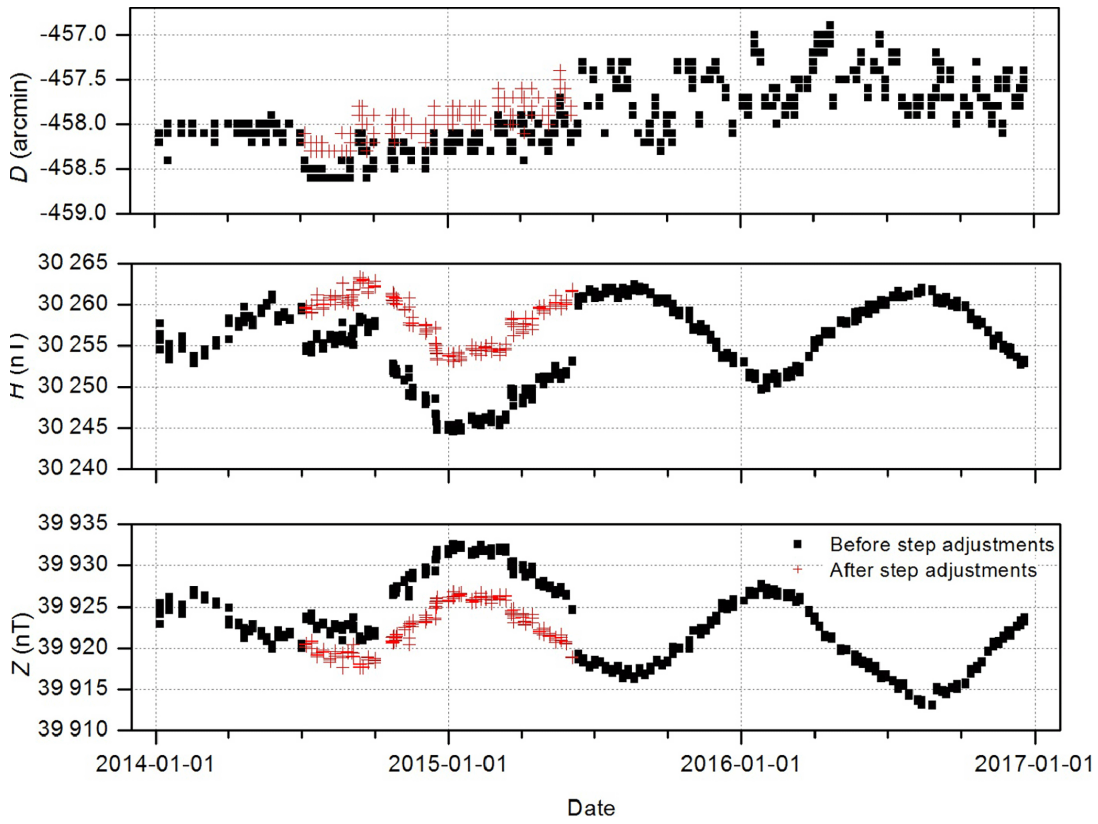

Figure 5. $D, H$ and $Z$ baselines before adjustment (solid rectangular) and after adjustment (cross) of steps.

tober 2014 to 4 June 2015. $D, H$ and $Z$ baselines after adjustments are presented in Fig. 5. The baseline shift in $H$ and $Z$ reduced to approximately $2 \mathrm{nT}$, while $D$ baselines show a noticeable improvement after the correction.

We also checked for the stability of the fluxgate sensor mounting. Some sensors in the DTU single-axis fluxgate magnetometers are reported to give unstable readings of the offset due to loose ferromagnetic cores (Pedersen and Matzka, 2012). The sensor instability can give a discrepancy in zero readings of absolute measurement. In order to check the loose-core problem in the single-axis fluxgate used at the CYG observatory, the sensor offset and the collimation angle from the $D$ and $I$ measurements were calculated. The sensor offset included the residual magnetism of the magnetometer 

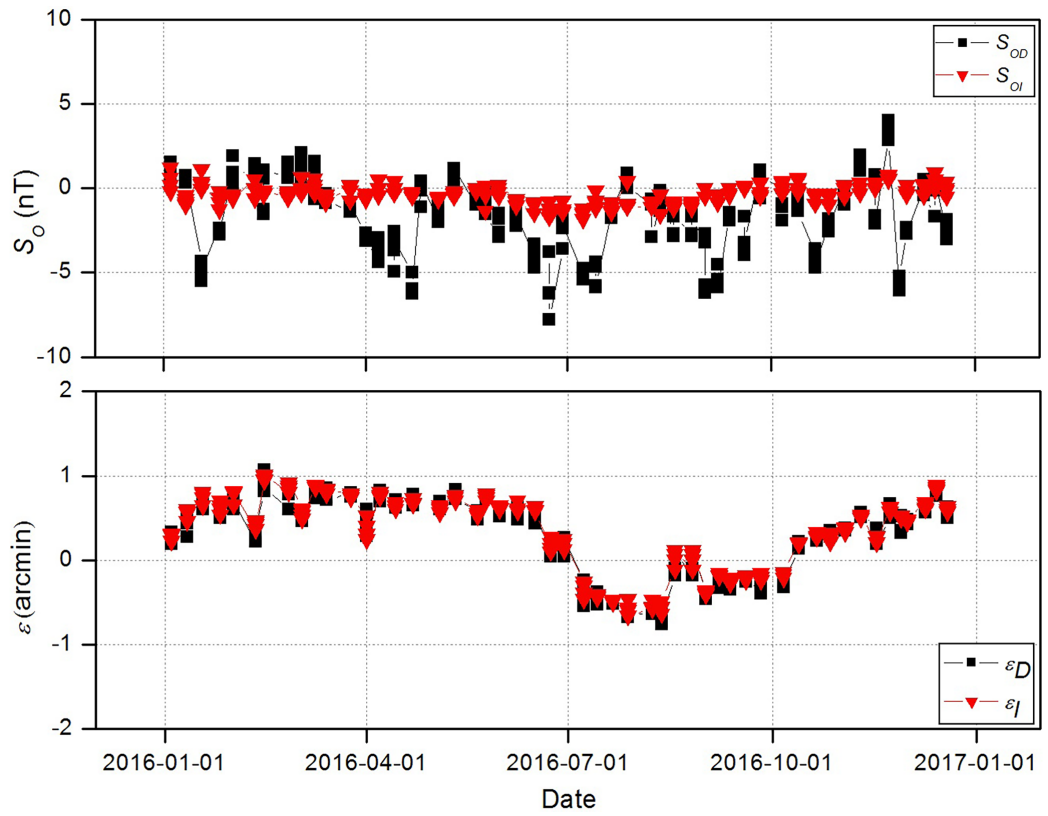

Figure 6. (Top) Sensor offset $S_{\mathrm{O} D}$ for the declination and $S_{\mathrm{O} I}$ for the inclination; (bottom) collimation angle $\varepsilon_{D}$ for the declination and $\varepsilon_{I}$ for the inclination.
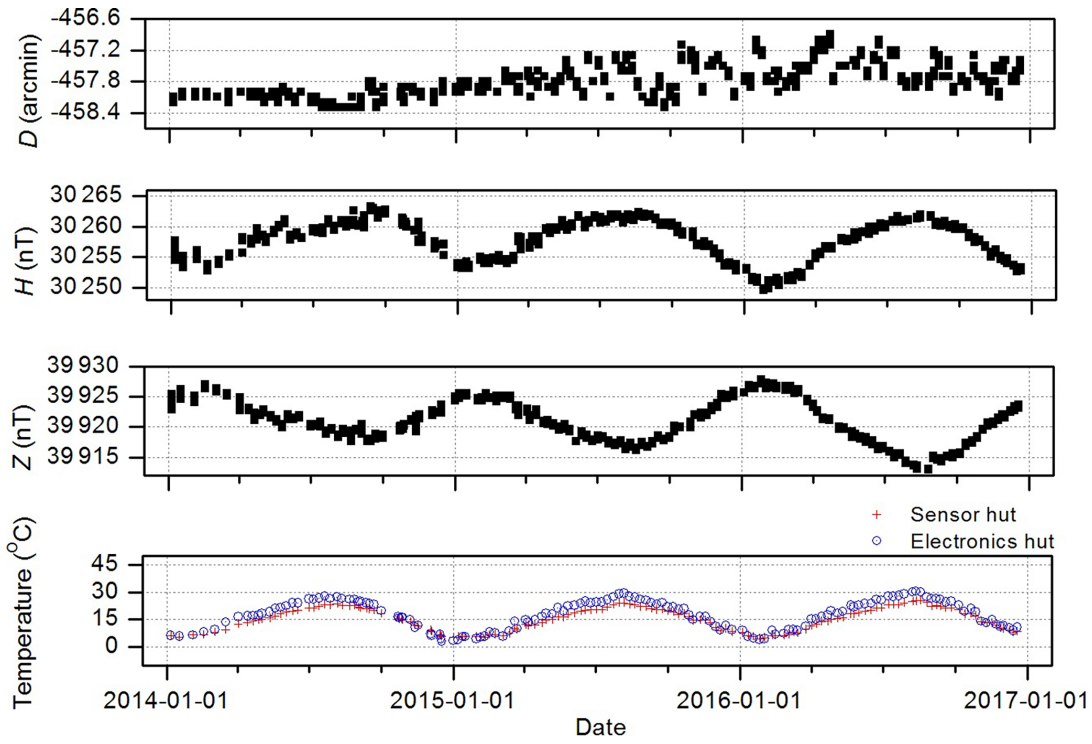

Figure 7. $D, H$ and $Z$ baselines and (lowest panel) temperature of the fluxgate magnetometer sensor and the electronic huts.

and the offset of the electronics calculated from the $D$ and the $I$ circle readings according to Eqs. (1) and (2) (Turbitt, 2004):

$$
\begin{aligned}
s_{\mathrm{O} D} & =H \sin [(\text { east down }+ \text { east up }- \text { west down } \\
& - \text { west up }) / 4], \\
s_{\mathrm{O} I} & =F \sin [(\text { south down }+ \text { south up }- \text { north down } \\
& - \text { north up) } / 4] .
\end{aligned}
$$

The collimation angle $\varepsilon$ is the angle between the measurement axis of the magnetometer and the optical axis of the telescope on a vertical plane. The angle is calculated from $D$ 


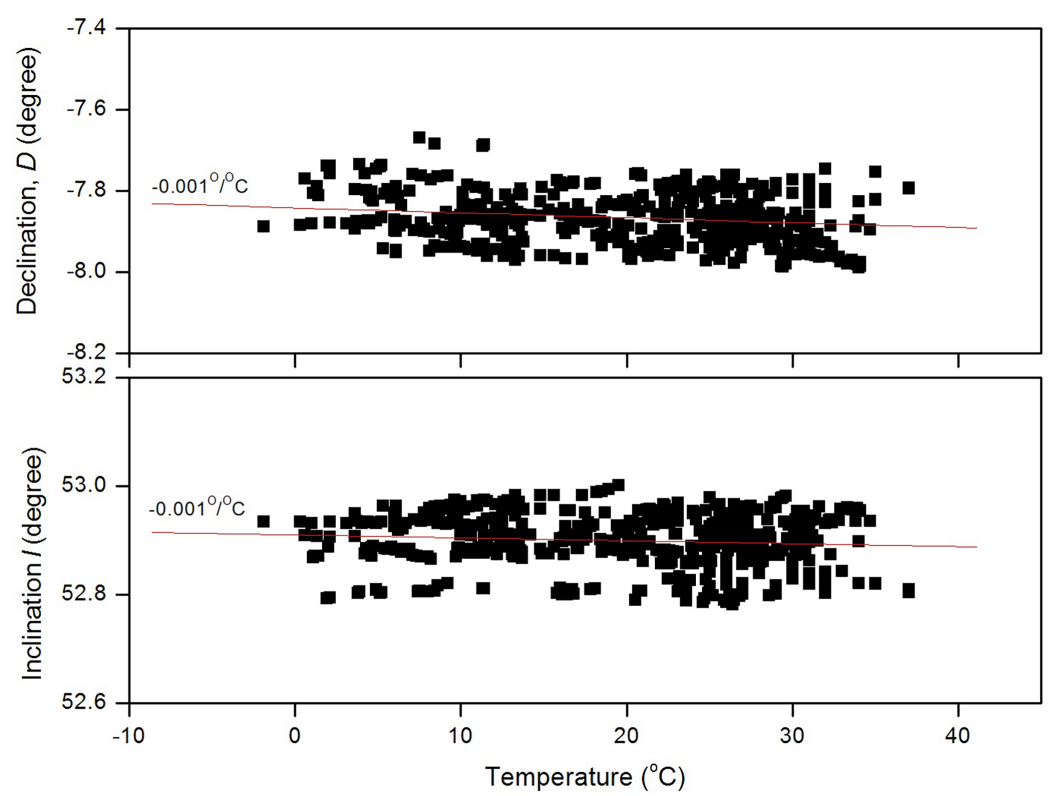

Figure 8. (Top) Declination and (bottom) inclination value plotted against temperature in the absolute-measurement hut.

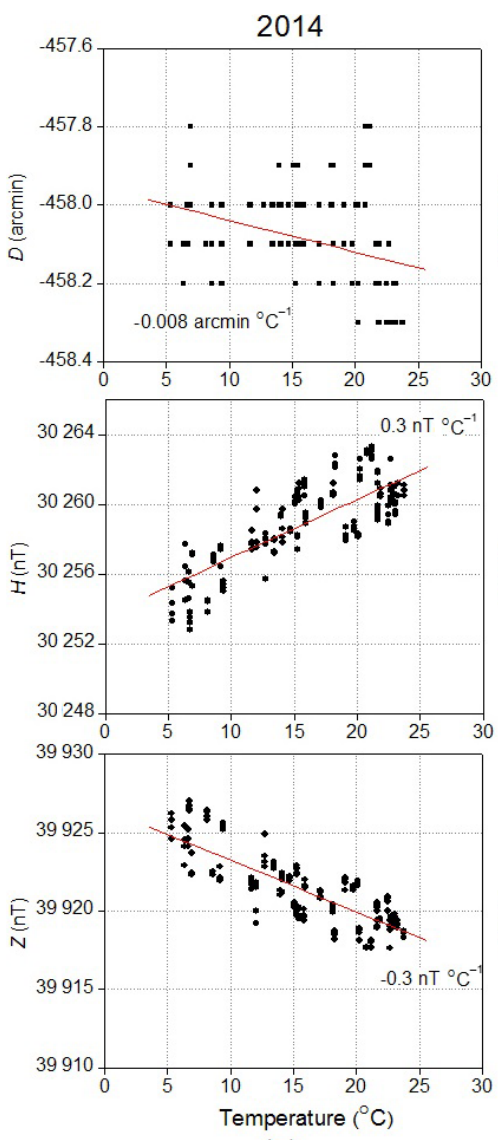

(a)
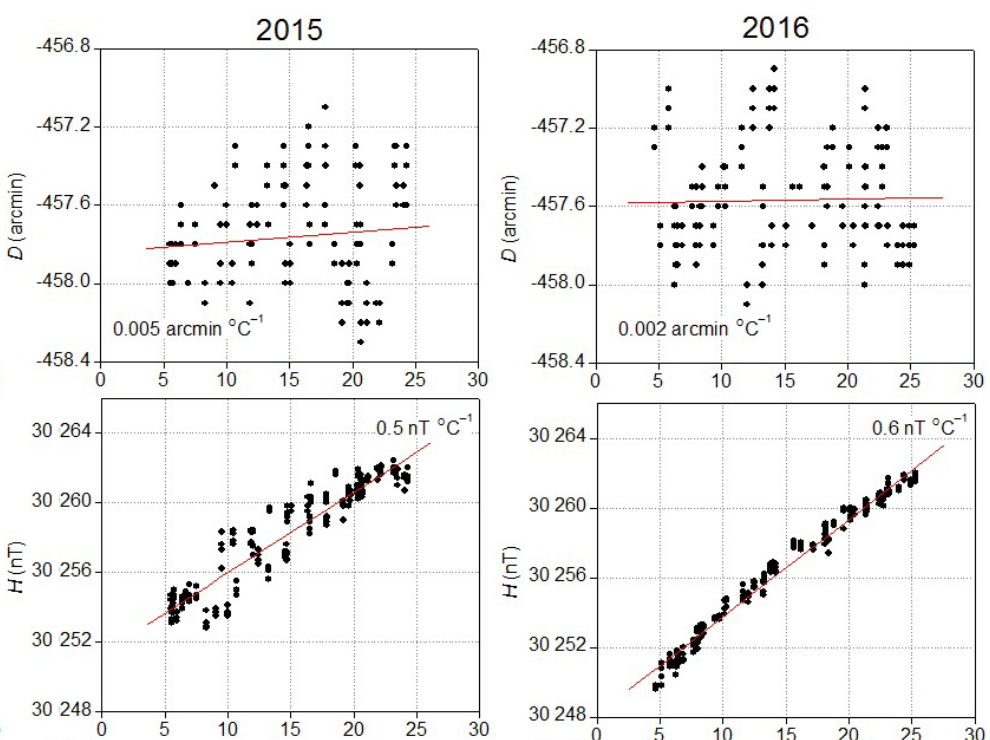

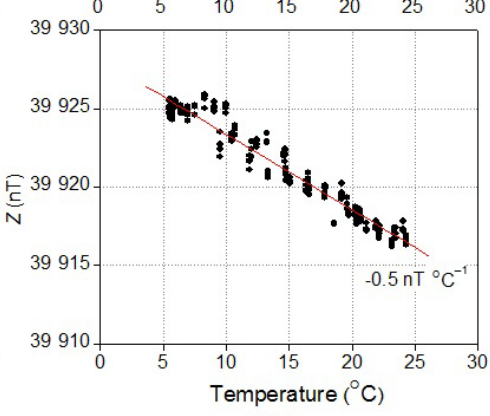

(b)

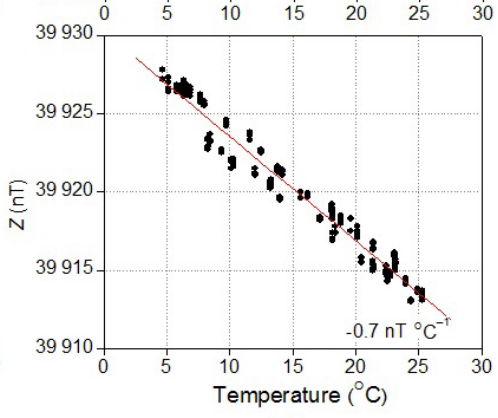

(c)

Figure 9. Temperature coefficient of $D, H$ and $Z$ baselines for (a) 2014, (b) 2015 and (c) 2016. 


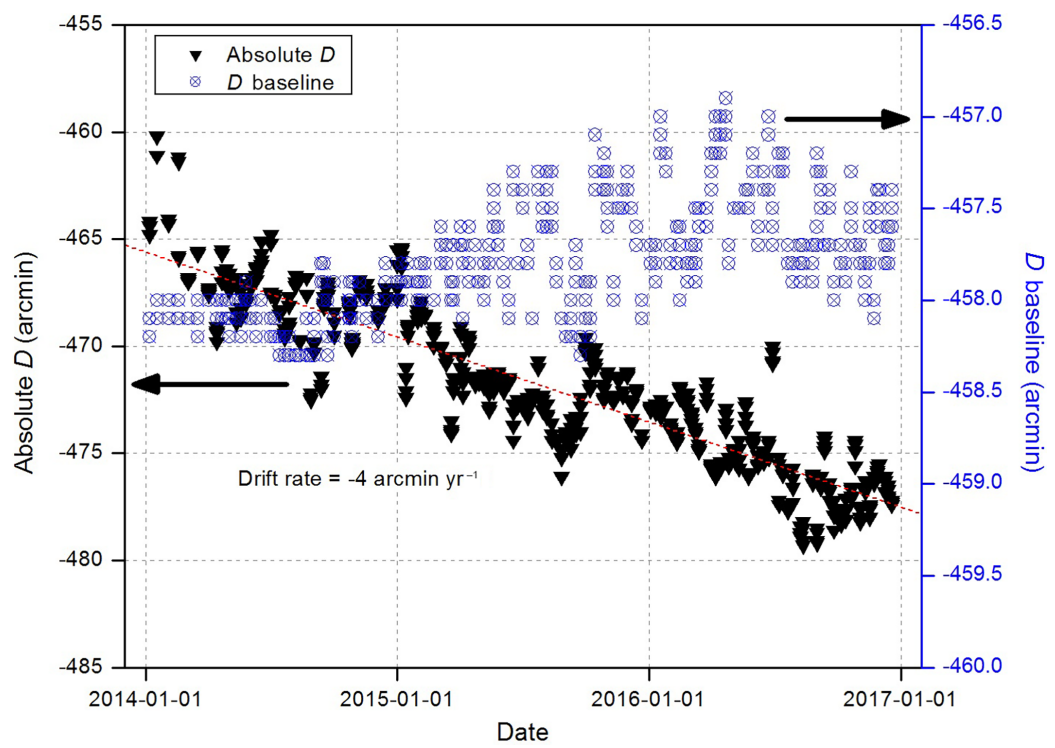

Figure 10. Comparison of $D$ baselines (circle with cross) and absolute $D$ (triangle) from 2014 to 2016 . The dashed line is the linear fit to the absolute measurement.

and $I$ circle readings as below:

$$
\begin{aligned}
\varepsilon_{D} & =(\text { west down }+ \text { east up }- \text { east down } \\
& \left.- \text { west up } \pm 360^{\circ}\right) /(4 \cdot \tan I), \\
\varepsilon_{I} & =(\text { north down }+ \text { south up }- \text { north up } \\
& - \text { South Down }) / 4,
\end{aligned}
$$

The result in Fig. 6 shows that the sensor offset $S_{\mathrm{O} D}$ and $S_{\mathrm{O} I}$ agreed within $10 \mathrm{nT}$ and that the collimation angles $\varepsilon_{D}$ and $\varepsilon_{I}$ are constant within \pm 1 arcmin. Neither the sensor offset $S_{\mathrm{O} D}$ and $S_{\mathrm{O} I}$ nor the collimation angle $\varepsilon_{D}$ and $\varepsilon_{I}$ shows large discrepancies that can cause an error in the absolute measurement. Thus, the analysis above implies that the sensors used at CYG are stable.

\section{Baseline variations}

Figure 7 presents the $D, H$ and $Z$ baselines and daily mean temperature in the fluxgate magnetometer sensor and electronics hut. The temperature effect on the fluxgate magnetometer measurement can be clearly seen on $H$ and $Z$ baselines, although the $D$ component does not show a clear relation to temperature changes. The fluxgate sensor and electronics experienced significant temperature swings, as much as $20^{\circ} \mathrm{C}$ annually. Daily temperature variations of $0.2-3{ }^{\circ} \mathrm{C}$ were observed in the sensor hut and of $0.6-5^{\circ} \mathrm{C}$ in the electronics hut. In order to check the temperature effect on the absolute instruments, the declination and inclination values as a function of temperature obtained in the absolutemeasurement hut during the observation are plotted in Fig. 8. Both $D$ and $I$ show a small change with the temperature rate of $-0.001 \%{ }^{\circ} \mathrm{C}$. We could assume that the temperature effect observed in baselines is mainly due to the fluxgate magnetometer.

Figure 9 presents the temperature-dependent variations of the $D, H$ and $Z$ baselines depicted as a function of temperature in the sensor hut from 2014 to 2016 and their calculated temperature coefficients. The baselines show an increasing amplitude with temperature, indicating that the fluxgate has a large temperature coefficient, mainly in the $H$ and $Z$ components. Csontos et al. (2007) proved that most of the fluxgate magnetometers have a large temperature coefficient, and their behaviour depends significantly on the amplitude of temperature change. The temperature coefficients of the $H$ baseline increased from $0.3 \mathrm{nT}^{\circ} \mathrm{C}^{-1}$ in 2014 to $0.6 \mathrm{nT}^{\circ} \mathrm{C}^{-1}$ in 2016 , whereas the temperature coefficients of the $Z$ baseline varied from $-0.3 \mathrm{nT}^{\circ} \mathrm{C}^{-1}$ in 2014 to $-0.7 \mathrm{nT}^{\circ} \mathrm{C}^{-1}$ in 2016 . Temperature influence on $D$ baselines is considerably low with respect to that of the $H$ and $Z$ baselines, and it changed from -0.008 arcmin ${ }^{\circ} \mathrm{C}^{-1}$ in 2014 to $0.002 \operatorname{arcmin}^{\circ} \mathrm{C}^{-1}$ in 2016 . This varying sensitivity in the temperature coefficient limits the possibility to determine a general correction factor for temperature effect. Hence, the use of a temperature-stabilized environment is the best way to achieve very accurate measurement (Csontos et al., 2007).

The corrected baselines in Fig. 7 show better stability with time. The dispersion of consecutive measurements is well less than $1 \mathrm{nT}$ in $H$ and $Z$ throughout the period, with standard deviation reduced by $30 \%$ in 2016 , indicating that the quality of the absolute measurement has improved over the period. Although $D$ baselines show a larger deviation in 2015 and 2016, the accuracy of absolute $D$ measurement improved as seen from the scatter of the data shown in Fig. 10. The standard deviation of dispersion reduced by $20 \%$ in 2015 
and 2016. The absolute $D$ also shows a decreasing value with time, in contrast with $D$ baselines. Comparison of the absolute $D$ values with the International Geomagnetic Reference Field (IGRF) model shows a similar trend and rate of change, which is approximately $-4 \operatorname{arcmin}_{\mathrm{yr}^{-1}}$ (dashed line in Fig. 10).

\section{Conslusion}

Variations of baselines produced by the CYG observatory from a period of 2014 to 2016 are analysed. Steps of more than $5 \mathrm{nT}$ were found in $H$ and $Z$ baselines, causing a baseline shift from July 2014 to June 2015. The installation of the LED light panels was identified as the reason for the jumps in the absolute measurement during this period. Steps are reduced to less than $5 \mathrm{nT}$ after adjustments of the baselines. Generally, the baselines produced by the CYG observatory comply with the INTERMAGNET standards, which shows the capability of CYG to produce high-quality data. The quality of the absolute measurement has improved with time as seen by the scatter of the data.

Temperature variation, ageing of electronic components, pier tilts etc. are known factors that can affect the long-term stability of baselines. The temperature effect was supposed to be a major reason for the large drift in the CYG baselines. Use of a temperature-stabilized environment is the best way to minimize the temperature effect of the fluxgate magnetometer and to achieve accurate measurements. Levelling and target readings; stability of fluxgate theodolite; magnetic cleanliness; etc. can affect the accuracy of the absolute measurement and should always be checked during observations to avoid unnecessary steps from occurring in the absolute measurement.

Data availability. The CYG observed baseline values are available in the Supplement.

\section{The Supplement related to this article is available online at https://doi.org/10.5194/gi-6-231-2017-supplement.}

Competing interests. The authors declare that they have no conflict of interest.

Acknowledgements. We would like to thank Orsi Baillie from the British Geological Survey for her continuous support and assistance in data processing. Special thanks to Tero Raita from the Sodankylä Geophysical Observatory for his time and helpful advice in checking the baselines. We would also like to thank the Kakioka Magnetic Observatory, Japan Meteorological Agency, for providing the KAK baseline data.
This work was funded by the Korea Meteorological Administration Research and Development Program under grant KMIPA2015-3030.

Edited by: Jean Rasson

Reviewed by: two anonymous referees

\section{References}

Clarke, E., Baillie, O., Reay, S. J., and Turbitt, C. W.: A method for the near real-time production of quasi-definitive magnetic observatory data, Earth Planets Space, 65, 1363-1374, 2013.

Csontos, A., Hegymegi, L., and Heilig, B.: Temperature Tests on Modern Magnetometers', Publs. Inst. Geophys. Pol. Acad. Sc. C, 99, 171-177, 2007.

Jankowski, J. and Sucksdorff, C.: Guide for Magnetic Measurements and Observatory Practice, International Association of Geomagnetism and Aeronomy, Warsaw, 1996,

McLean, S., Macmillan, S., Maus, S., Lesur, V., Thomson, A., and Dater, D.: The US/UK World Magnetic Model for 2005-2010, NOAA Technical Report NESDIS/NGDC-1, USA, 2004.

Park, P. G., Kim, W. S., Kim, M. S., and Shifrin, V. Ya.: Measurement and Analysis of Earth's Magnetic Field based on LowMagnetic Field Standards, CPEM Conference Digest, 29th Conference on Precision Electromagnetic Measurements (CPEM), IEEE, 358-359, 2014.

Pedersen, L. W. and Matzka, J.: Detection of Mechanical Instability in DI-Fluxgate Sensors, in: Proceedings of the XVth IAGA Workshop on Geomagnetic Observatory Instruments, edited by: Hejda, P., Chulliat, A., and Catalan, M., Data Acquisition, and Processing: Extended Abstract Volume, 61-64, 2012.

Reda, J., Fouassier, D., Isac, A., Linthe, H. J., Matzka, J., and Turbitt, C. W.: Improvements in geomagnetic observatory data quality, in: Geomagnetic Observations and Models, edited by: Mandea, M. and Korte, M., IAGA Special Sopron Book Series, 5, 127-148, https://doi.org/10.1007/978-90-481-9858-0_7, 2011.

Shifrin, V. Y., Khorev, V. N., Kalabin, V. N., and Park, P. G.: Experimental estimation of the accuracy of modern scalar quantum magnetometers in measurements of the Earth's magnetic field, Phys. Earth Planet. In., 166, 147-152, 2008.

St. Louis, B. (Ed.): INTERMAGNET Technical Reference Manual, Version 4.6, available at: http://www.intermagnet. org/publication-software/technicalsoft-eng.php (last access: 28 November 2014), 2012.

Turbitt, C. W.: Procedure for Determining Instantaneous GDAS Variometer Baselines from Absolute Observations, British Geological Survey, Edinburgh UK, 2004. 